\title{
Electronic properties of organic semiconductor blends: Ambipolar mixtures of phthalocyanine and fullerene
}

Cite as: Appl. Phys. Lett. 90, 212112 (2007); https://doi.org/10.1063/1.2742640

Submitted: 07 March 2007 . Accepted: 01 May 2007 . Published Online: 24 May 2007

Andreas Opitz, Markus Bronner, Wolfgang Brütting, Marcel Himmerlich, Juergen A. Schaefer, and Stefan Krischok

\section{ARTICLES YOU MAY BE INTERESTED IN}

Two-layer organic photovoltaic cell

Applied Physics Letters 48, 183 (1986); https://doi.org/10.1063/1.96937

Ambipolar charge carrier transport in mixed organic layers of phthalocyanine and fullerene Journal of Applied Physics 101, 063709 (2007); https://doi.org/10.1063/1.2436836

Small molecular weight organic thin-film photodetectors and solar cells

Journal of Applied Physics 93, 3693 (2003); https://doi.org/10.1063/1.1534621

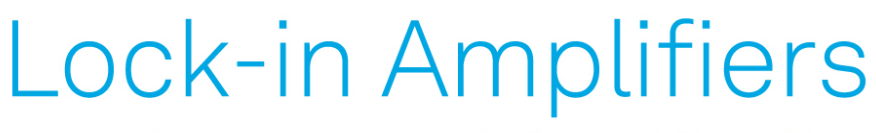

... and more, from DC to $600 \mathrm{MHz}$

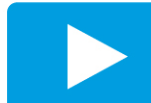

Watch 


\title{
Electronic properties of organic semiconductor blends: Ambipolar mixtures of phthalocyanine and fullerene
}

\author{
Andreas Opitz, ${ }^{\text {a) }}$ Markus Bronner, and Wolfgang Brütting \\ Institute of Physics, University of Augsburg, 86135 Augsburg, Germany \\ Marcel Himmerlich, Juergen A. Schaefer, and Stefan Krischok \\ Institute of Physics and Institute of Micro- and Nanotechnologies, Technical University Ilmenau, 98684 \\ Ilmenau, Germany
}

(Received 7 March 2007; accepted 1 May 2007; published online 24 May 2007)

\begin{abstract}
Mixtures of electron and hole transporting organic materials are widely used for ambipolar organic field-effect transistors and photovoltaic cells. One particular material system used in both device types is a blend of $n$-conducting fullerene and $p$-conducting copper-phthalocyanine. The electronic properties of these blends were analyzed by x-ray and ultraviolet photoelectron spectroscopy in dependence on the mixing ratio. The energies of the highest occupied molecular orbitals, the core levels, and the vacuum level are found to vary linearly with the mixing ratio. This energy shift is related to a common work function in the molecular blends. (C) 2007 American Institute of Physics. [DOI: $10.1063 / 1.2742640]$
\end{abstract}

Multilayered organic systems are used in light emitting diodes and in photovoltaic cells for spatially separated electron and hole transports. However, an increase of efficiency in organic photovoltaic cells (OPVCs) is achieved by using mixed layers of the electron and hole conducting materials. ${ }^{1,2}$ This concept of mixed layers is also used in organic fieldeffect transistors (OFETs) and leads to devices with ambipolar transport characteristics. ${ }^{3,4}$ For the description of device properties, knowledge about the electronic structure of the materials is important, but up to date only neat materials were analyzed in detail. To understand the ambipolar transport characteristics, these studies need to be extended to mixed systems.

The scope of this study is the analysis of the electronic structure of copper-phthalocyanine $(\mathrm{CuPc})$ and buckminsterfullerene $\left(C_{60}\right)$ blends which have recently been employed as donor-acceptor systems for both OFETs and OPVCs. ${ }^{4,5}$ Both materials are coevaporated using two effusion cells with different evaporation rates to achieve the required mixing ratios. All organic films have a nominal film thickness of $25 \mathrm{~nm}$ recorded by a quartz crystal microbalance. As substrate we use gold films (thickness of about $100 \mathrm{~nm}$ ) which are thermally evaporated onto oxidized $\mathrm{Si}$ wafers and pretreated in an oxygen plasma prior to the deposition of the organic materials. The surface properties of the films were characterized using x-ray photoelectron spectroscopy (XPS) and ultraviolet photoelectron spectroscopy (UPS) by employing monochromated $\mathrm{Al} K \alpha$ radiation $(h \nu=1486.7 \mathrm{eV})$ for the measurement of the core levels as well as ultraviolet radiation [He I $(h \nu=21.2 \mathrm{eV})$ and $\mathrm{He}$ II $(h \nu=40.8 \mathrm{eV})]$ for an analysis of the occupied states near the Fermi level. For the measurement of the secondary electron cutoff to exactly determine the sample work function, an additional bias ( -2 and $-5 \mathrm{~V}$ ) was applied to the sample.

It should be noted that transporting the gold films through ambient air after the oxygen plasma treatment leads to the formation of a conductive hydrocarbon layer. ${ }^{6}$ The

\footnotetext{
a) Author to whom correspondence should be addressed; electronic mail:
} andreas.opitz@physik.uni-augsburg.de work function of gold covered by this hydrocarbon layer is determined by photoelectron spectroscopy to be $4.2 \mathrm{eV}$, whereas the underlying gold substrate exhibits a work function of $5.4 \mathrm{eV}$ after removal of the adsorbed species by $\mathrm{Ar}^{+}$ ion bombardment.

Figure 1 shows the important results of the XPS and UPS measurements. The CuPc $\mathrm{C} 1 s$ spectrum contains the $\mathrm{C}-\mathrm{C}$ bond $\left(E_{B}=284.2 \mathrm{eV}\right.$, indicated by small ticks) and the chemically shifted $\mathrm{C}-\mathrm{N}\left(E_{B}=285.6 \mathrm{eV}\right)$ bond, as well as their $\pi-\pi^{*}$ satellites. For $\mathrm{C}_{60}$ only one pronounced feature is visible at $E_{B}=284.7 \mathrm{eV}$. Both spectra are in agreement with published results on pristine materials. ${ }^{7,8}$ The composition of the blends and the CuPc and $\mathrm{C}_{60}$ related $\mathrm{C} 1 s$ peak positions are determined by fitting the spectra of the mixed films with Gauss-Lorentz functions using the shape of the neat $\mathrm{C}_{60}$ and $\mathrm{CuPc}$ core levels. As shown in Fig. 1(a), the energetic positions of the $\mathrm{C} 1 s$ features are slightly shifting upon addition of $\mathrm{C}_{60}$. The $\mathrm{N} 1 s$ spectra (not shown) were also analyzed for the peak position and found to behave in a similar manner.

The electronic structure of the occupied states near the Fermi level (measured using He II radiation) is shown in Fig. 1(b). The maxima of the highest occupied molecular orbital (HOMO) levels of the neat materials are indicated by small ticks. Whereas the CuPc HOMO level is clearly separated for all mixed films, the HOMO level of $\mathrm{C}_{60}$ is only visible in films with concentrations of $77 \%$ and $100 \%$. For smaller amounts of $\mathrm{C}_{60}$ in the films the HOMO level is present only as a shoulder in the occupied molecular orbitals of the CuPc. Again, the spectra can be described as a linear superposition of neat material spectra if a shift of the energetic position is included.

The work function of the blend films was determined from the secondary electron cutoff in the He I spectra with applied bias [see Fig. 1(c)]. As for the core and the HOMO levels an energy shift of the secondary electron cutoff with the film composition is present.

The graph in Fig. 2 shows a comparison of the obtained vacuum levels of the organic films and the substrate $\left(E_{\mathrm{vac}}^{\mathrm{org}}\right.$ and $E_{\mathrm{vac}}^{\mathrm{sub}}$ ), of the HOMO levels $\left(E_{\mathrm{HOMO}}^{\text {org }}\right)$ (defined as the HOMO edge near the Fermi level), as well as of the $\mathrm{C} 1 s$ and 


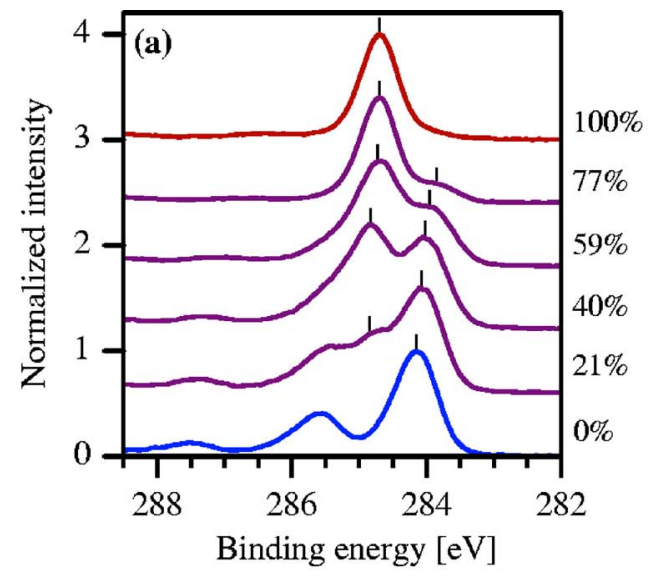

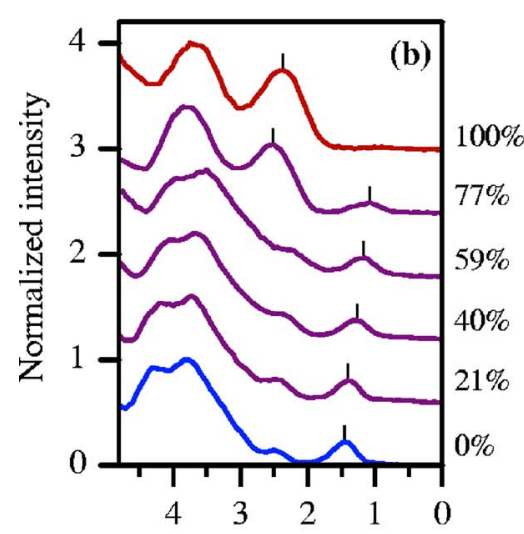

Binding energy $[\mathrm{eV}]$

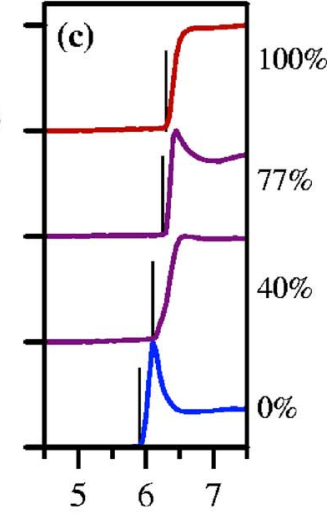

Kinetic energy $[\mathrm{eV}]$
FIG. 1. (Color online) XPS and UPS measurements of neat and different mixed films of $\mathrm{C}_{60}$ and CuPc: (a) $\mathrm{C} 1 \mathrm{~s}$ spectra (excitation: monochromated $\mathrm{Al} K \alpha$ ), (b) HOMO levels (excitation: He II), and (c) secondary electron cutoff (excitation: He I and $V_{\text {bias }}=-2 \mathrm{~V}$ ). The numbers on the right side of the diagrams give the $\mathrm{C}_{60}$ concentration as determined from the measurements shown in part (a).

$\mathrm{N} 1 s$ core levels $\left(E_{\mathrm{C} 1 s}^{\mathrm{org}}\right.$ and $E_{\mathrm{N} 1 s}^{\mathrm{org}}$ ) depending on the mixing ratio. The ionization energies of the neat films are $6.2 \mathrm{eV}$ for $\mathrm{C}_{60}$ and $5.0 \mathrm{eV}$ for $\mathrm{CuPc}$ in agreement with the literature ${ }^{9}$ and the difference of the work function amounts to about $0.5 \mathrm{eV}$.

It is remarkable that the secondary electron cutoffs used to determine the work function of all mixtures were sharp and did not show a double step that would reflect two different local surface potentials, ${ }^{10}$ although the films have a nanocrystalline morphology as determined by atomic force microscopy. ${ }^{4}$ Thus, the blends show a common vacuum level which shifts linearly with the concentration between the work functions of the neat materials given by $\Phi_{\mathrm{CuPc}}=3.8 \mathrm{eV}$ and $\Phi_{\mathrm{C}_{60}}=4.3 \mathrm{eV}$. The linear change of the work function in our studies suggests that $\mathrm{CuPc} / \mathrm{C}_{60}$ mixtures are electronically noninteracting (unless they are optically excited). In contrast, a nonlinear change of the work function was found for doping organic semiconductors with strong acceptors or donors ${ }^{11,12}$ where a charge transfer occurs already in the dark.

Taken all results together, there is no evidence for a ground state charge transfer in blends of $\mathrm{CuPc}$ and $\mathrm{C}_{60}$. The core level spectra can be described solely with the scaled features of the neat materials. Also the occupied molecular orbitals do not show additional structures unknown from the neat materials. ${ }^{9,13}$ Especially the HOMO level of $\mathrm{CuPc}$ is

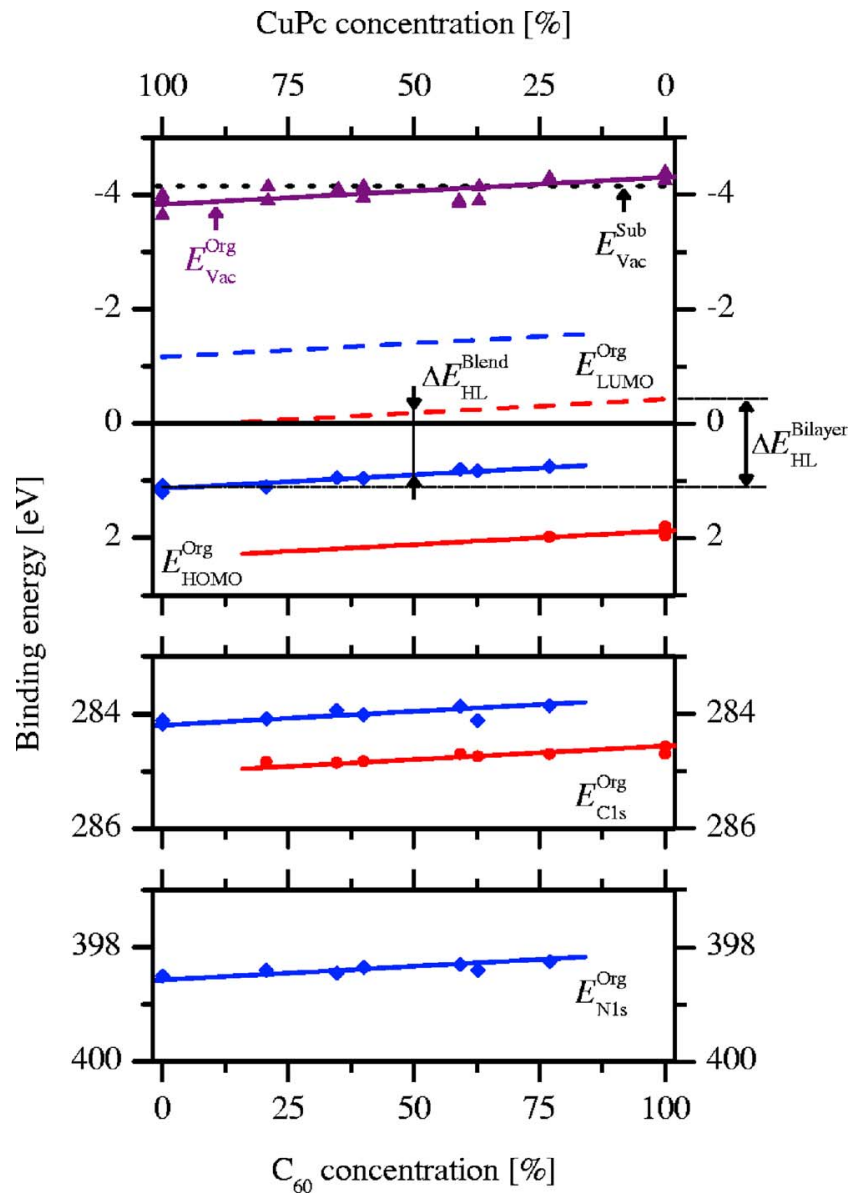

FIG. 2. (Color online) Positions of the energy levels (vacuum level, high energy edge of the HOMO level, and $\mathrm{C} 1 s$ and $\mathrm{N} 1 s$ core levels) depending on the $\mathrm{C}_{60} / \mathrm{CuPc}$ mixing ratio. The and the symbols represent the measured values for $\mathrm{C}_{60}$ and $\mathrm{CuPc}$ related levels, respectively, and the $\boldsymbol{\Delta}$ symbols the measured work functions. The (_- lines are linear fits of the corresponding measured values. The constant $(.$.$) is the vacuum energy$ of the conductive hydrocarbon layer on gold. The (- - -) lines are the calculated LUMO levels.

always well separated and does not tend to mix with other orbitals. Although the $\mathrm{HOMO}$ level of $\mathrm{C}_{60}$ lies in the range of other occupied levels of the CuPc, the spectra of the mixed films can be described as a linear superposition of the spectra of the neat materials. ${ }^{14}$ The analysis of the difference between the occupied levels (core and HOMO levels) and the vacuum level shows for all compositions a constant ionization energy. This is manifested in identical shifts of the core levels, the HOMO levels, and the vacuum level with the concentration of the blends. Thus the molecular levels are simply following the change of the common vacuum energy.

As already mentioned, blends of $\mathrm{CuPc}$ and $\mathrm{C}_{60}$ are frequently used in photovoltaic cells where a photoinduced charge transfer with $\mathrm{CuPc}$ acting as electron donor and $\mathrm{C}_{60}$ as electron acceptor, respectively, occurs. Their open circuit voltage is expected to depend on the gap between the lowest unoccupied molecular orbital (LUMO) of the acceptor and the HOMO of the donor. ${ }^{2}$ Therefore Fig. 2 also contains the calculated LUMO levels assuming a transport gap of $2.3 \mathrm{eV}$ for both materials ${ }^{15,16}$ independent of the mixing ratio. The assumption of a constant transport gap is supported by results of absorption measurements ${ }^{17}$ and corresponds to the independence of the ionization potential on the mixing ratio and consequently to the existence of an unchanged electron 
affinity of the blend system. From these levels one expects a reduction of the HOMO-LUMO gap $\Delta_{\mathrm{HL}}=E_{\mathrm{HOMO}}^{\mathrm{CuPc}}-E_{\mathrm{LUMO}}^{\mathrm{C}_{60}}$ by about $0.5 \mathrm{eV}$ in going from a heterolayer structure $\left(\Delta_{\mathrm{HL}}^{\text {Bilayer }}\right)$ to a bulk-heterojunction device $\left(\Delta_{\mathrm{HL}}^{\text {Blend }}\right)$. Thereby, as indicated in Fig. 2, the HOMO-LUMO gap of a bilayer system $(\sim 1.6 \mathrm{eV})$ is given by the respective levels of the neat materials in agreement with Ref. 9, whereas the value for the mixtures is found to be smaller $(\sim 1.1 \mathrm{eV})$ and independent of the composition of the blends. Qualitatively, a reduction of the open circuit voltage in bulk-heterojunction devices has been observed in the $\mathrm{CuPc} / \mathrm{C}_{60}$ system at high illumination intensities, 5 as well as for other material combinations using small molecules ${ }^{18}$ or polymers. ${ }^{19}$

Additionally, as the barrier for charge carrier injection is determined by the difference of the Fermi level of the metallic electrode and the LUMO or HOMO level of the electron and hole transporting organic materials, respectively, these results are also important for other organic devices, such as OFETs. Neglecting interface effects (interface dipole and band bending) the injection barrier for both electrons and holes should be reduced in ambipolar blend systems; however, as the diffusion limited injection current is proportional to the charge carrier mobility, ${ }^{20}$ the barrier lowering might be overcompensated by a decreased mobility in blend systems. ${ }^{4}$ Further investigations will be necessary to quantify both concurring effects.

In conclusion, the HOMO levels, the core levels and the vacuum level of the $\mathrm{CuPc} / \mathrm{C}_{60}$ blends shift almost linearly with the mixing ratio. Thereby a common vacuum level exists in these blends, which varies between the vacuum levels of the neat materials. The HOMO and the core levels show the molecular features and follow the change of the vacuum level. The observed energy level shifts are expected to have implications both for OFETs and OPVCs.
This work was supported by the Deutsche Forschungsgemeinschaft through Sonderforschungsbereich 484 and Schwerpunktprogramm 1121.

${ }^{1}$ J. Rostalski and D. Meissner, Sol. Energy Mater. Sol. Cells 61, 87 (2000). ${ }^{2}$ C. J. Brabec, V. Dyakonov, J. Parisi, and N. S. Sariciftci, Organic Photovoltaics (Springer, Berlin, 2003).

${ }^{3}$ E. J. Meijer, D. M. De Leeuw, S. Setayesh, E. Van Veenendaal, B. H. Huisman, P. W. M. Blom, J. C. Hummelen, U. Scherf, and T. M. Klapwijk, Nat. Mater. 2, 678 (2003).

${ }^{4}$ A. Opitz, M. Bronner, and W. Brütting, J. Appl. Phys. 101, 063709 (2007).

${ }^{5}$ J. G. Xue, B. P. Rand, S. Uchida, and S. R. Forrest, J. Appl. Phys. 98, 124903 (2005).

${ }^{6}$ S. Rentenberger, A. Vollmer, E. Zojer, R. Schennach, and N. Koch, J. Appl. Phys. 100, 053701 (2006).

${ }^{7}$ M. Knupfer and H. Peisert, Phys. Status Solidi A 201, 1055 (2004).

${ }^{8}$ T. R. Ohno, Y. Chen, S. E. Harvey, G. H. Kroll, J. H. Weaver, R. E. Haufler, and R. E. Smalley, Phys. Rev. B 44, 13747 (1991).

${ }^{9}$ O. V. Molodtsova and M. Knupfer, J. Appl. Phys. 99, 053704 (2006).

${ }^{10}$ G. Koller, B. Winter, M. Oehzelt, J. Ivanco, F. P. Netzer, and M. G. Ramsey, Org. Electron. 8, 63 (2007).

${ }^{11}$ W. Y. Gao and A. Kahn, J. Phys.: Condens. Matter 15, S2757 (2003).

${ }^{12}$ S. Tanaka, E. Kawabe, K. Kanai, T. Iwahashi, T. Nishi, Y. Ouchi, and K. Seki, J. Electron Spectrosc. Relat. Phenom. 144-147, 533 (2005).

${ }^{13}$ L. Lozzi, V. Granato, S. Picozzi, M. Simeoni, S. La Rosa, B. Delly, and S. Santucci, J. Vac. Sci. Technol. A 24, 1668 (2006).

${ }^{14}$ N. Koch, I. Salzmann, R. L. Johnson, J. Pflaum, R. Friedlein, and J. P. Rabe, Org. Electron. 7, 537 (2006).

${ }^{15}$ R. W. Lof, M. A. Vanveenendaal, B. Koopmans, H. T. Jonkman, and G. A. Sawatzky, Phys. Rev. Lett. 68, 3924 (1992).

${ }^{16}$ I. Hill, A. Kahn, Z. Soos, and R. Pascal, Chem. Phys. Lett. 327, 181 (2000).

${ }^{17}$ B. P. Rand, J. G. Xue, S. Uchida, and S. R. Forrest, J. Appl. Phys. 98, 124902 (2005).

${ }^{18}$ C. Melzer, V. V. Krasnikov, and G. Hadziioannou, J. Polym. Sci., Part B: Polym. Phys. 41, 2665 (2003).

${ }^{19}$ Y. Hayashi, I. Yamada, S. Takagi, A. Takasu, T. Soga, and T. Jimbo, Jpn. J. Appl. Phys., Part 1 44, 1296 (2005).

${ }^{20}$ S. Sze, Physics of Semiconductor Devices (Wiley, New York, 1982). 